\title{
THE DEVELOPMENT OF THE COMPUTER-AIDED DESIGN SYSTEM FOR PRODUCTION PROCESSES OF COMPONENT PART MACHINING FOR SINGLE-PIECE PRODUCTION AND REPAIR CONDITIONS
}

\author{
Gulnara Zhetessova, Vasiliy Yurchenko, Tatyana Nikonova*, Olga Zharkevich \\ Karaganda state technical university, Kazakhstan
}

The given article provides material on the development of the CADS applied for production processes of component part machining for single-piece production and repair conditions. The article describes technique used to organize a data flow and hose methods of machining which cannot be applied under these conditions of machining. This research uses graph theory. The article describes the developed algorithm for machining planning of details. A mathematical model of transitions in the technological process for the processing of parts has been obtained. The developed design CADS reduces the number of errors in the development of production processes. The article describes database for machining planning. In order to form input data, knowledge of production processes of analogous machine parts or products production is not required. Thus developed models of system determines of machine-part production stages. This system gives schemes of its basing, a set of bases and an order of their preparation and forming of schemes of technological dimensional chains. This system counts and recounts interoperational dimensions. Developed models and system allows to increase amount of output information to the level of operational flow charts. The designed system gives the chance of its use in modern multiproduct. This system is universal, multifunctional.

Key words: mechanical engineering, computer-aided design, technological process, computer modeling

\section{INTRODUCTION}

The type of production of modern machine-building enterprises is generally single, small-scale and in an exceptional case medium scale. In addition, there is a big number of repair shops. It is known that in case of such organization of production the nomenclature of products is extensive, terms of finished product output are limited thus time for technological preparation of production is tight, hereby the process of development of production processes is very labor-consuming and connected with the emergence of a large number of errors. Terms of design of production processes for component parts of "Axis" type take up to 5-7 days, for component parts of "Case" type take from 7 to 14 days.

The main difficulty of manual design is impossibility of unambiguous solution making at the certain level, as the values of criteria for the choice of a particular element of production process will become known only at the next design stages, and often at the very end of design.

Ambiguity of the choice of certain solutions at intermediate design phases results in need for development of a large number of production process variants, which can be estimated only at the end of designing. At the same time, it is necessary to consider a huge number of criteria for objective assessment of the received variants. Thereby it is recommended to carry out final but already subjective choice of optimum production process at least of three most suitable solutions based on technical and economic assessment of the final project. As the variants of production processes have a huge number of data, the technologist with high probability will make the incorrect solution leading at best to adjustment of technology during the process of production, and at worst to defective products and heavy losses, both in material and monetary terms. Therefore, to make final decisions highly skilled technologists with wide experience are required, that is a huge problem for machine-building enterprises in connection with their deficiency or absence in general today. As the result, production processes are developed at the level of route technology in simplified form, without detailed study. In most cases the only variant, which is not always the best, is developed. At the same time in technological documentation there are no data necessary for technological preparation of production, that imposes the heavy responsibility on engineering and production workers, along with machine operators who are also poorly qualified today. All this leads to emergence of considerable time and material costs. If to consider the arising problems in case of component part production for mining machines, which is characteristic of Karaganda region (one of the largest industrial regions of the Republic of Kazakhstan), in addition to the specified reasons, they arise also due to heavy load and dimensions of these parts, and additional requirements imposed proceeding from severe conditions of operation. 


\section{AUTOMATION OF TECHNOLOGICAL PREPARATION OF PRODUCTION}

One of the ways out of the current situation is automation of technological preparation of production and, in particular, development and application of computer-aided design systems for production processes. Today there is a rather large number of such systems from various developers. The main disadvantage of such automated systems is that it is necessary to analyze and adjust results of designing of production processes according to a specific current situation at the place of production. The current situation is considerably connected with the fact that in the theory and methodology of process design there is a big number of recommendations on application of this or that technology solution which cannot be formalized and is applied only to specific cases. The vast majority of the existing CADs are constructed at best on the use of prototype solutions, and often the production engineer makes decisions himself, based on the personal experience or experience developed by manufacturer. Formalization of process of designing and technology, which will be a basis of the system of design automation, will give a chance of production process design for various products, including new, not produced before. To automate the CAD process, the design features of the CAD model are transferred into manufacturing features [1].Thus, the creation of automation system of technological preparation of production integrating various on nature and content tasks of machine-building technology is an urgent task.

The structure of a design technique of production process (PP) is presented in the Figure 1, and in the graph form in the Figure 2. The basis for this technique is a principle of feasibility of current transition, namely, implementation of characteristics, which need to be received at the given (current) transition. Herewith, practicing this method it is necessary to remove an overlap, which consists of characteristics of the previous and given method of machining. It is also considered that this overlap can be removed for several transitions, setting various cutting depth according to technological conditions of machining.

The following elements form characteristics of machining methods: dimensions of the machined surface, quality class reached by the given method, roughness, accuracy and values of spatial displacements of a surface, accuracy and values of spatial displacements of surface placement, depth of a defective layer, and cutting depth, which can be set for removal of over dimension.

All these characteristics correspond to the maximum accuracy values (the minimum deviation values) which can be received using the given tool, equipment, and the cutting modes. The description is done to obtain the maximum possible quantity of plan versions for machining with the following adjustment at the stages of tool choice, implement, and equipment. Herewith machining overlaps will be recalculated and cutting depth will be set

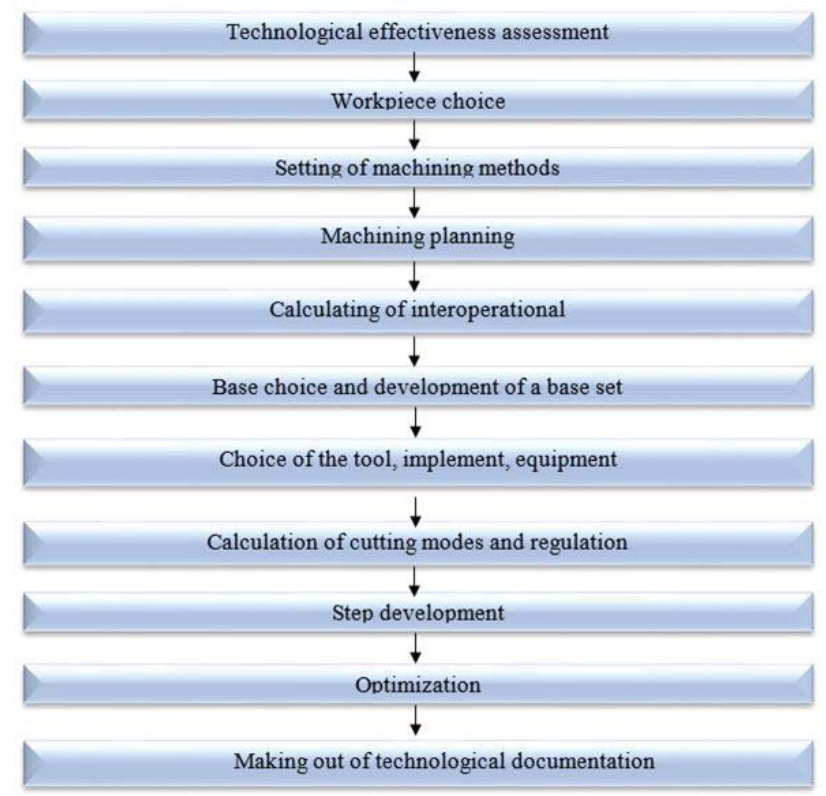

Figure 1: The structure of a design technique of production process.

according to characteristics of the tool, implement, and equipment.

The use of machining methods, which characteristics contain the maximum accuracy of machining, is explained by the fact that when machining workpiece surface by the same method, it is possible to use the tool of various design, hence it is possible to get various accuracy of machining, roughness, depth of a defective layer. The binding of characteristics of machining methods to characteristics of a specific tool will lead to significant and unjustified increase in amount of information. This postulate can be also applied to the other PP elements (implements, machines). The use of specific characteristics of PP elements is impossible because at this stage bases, sets of bases for machining of specific surfaces are unknown, as a type of base, its accuracy and its role in a set of bases is used for the choice of a tool, implement, and a machine.

If to make out a set of variants containing characteristics of the specific PP elements, and the sets of bases made out consequently will serve as criteria, then it will lead too to a big increase in information amount as characteristics of the PP elements contain a lot of information. Thus, the choice of bases and making out of a set of bases need to be made after machining planning.

Such technique is used to organize a data flow and to cut off those methods of machining which cannot be applied under these conditions of machining. The organization of a data flow, namely, machining planning and its partitioning into machining stages, is necessary for a task of base preparation.

A name of a machining stage is purely conditional here and corresponds actually not to a machining stage but to a particular quality class of a transition. The name is given for fuller understanding of a process of design 
which is applied in the manual mode, hence it is purely psychological. Setting of a machining stage in compliance with a particular quality class of machining is made because it is unknown on what quality class (transition) it is necessary to raise a quality class of a basic surface for machining of a particular surface.

According to such approach, such names as rough, finish, smooth machining will not be used, the name "a stage of the eighth quality class" will be used instead. Besides, it will correspond to partitioning into stages for non mechanical operations, e.g. a stage of heat treatment, where the stage corresponds to a particular method of machining. The partitioning into stages applied at manual design is used for simplification of perception of design process and does not reflect objective production processes, which means that it has a subjective character.
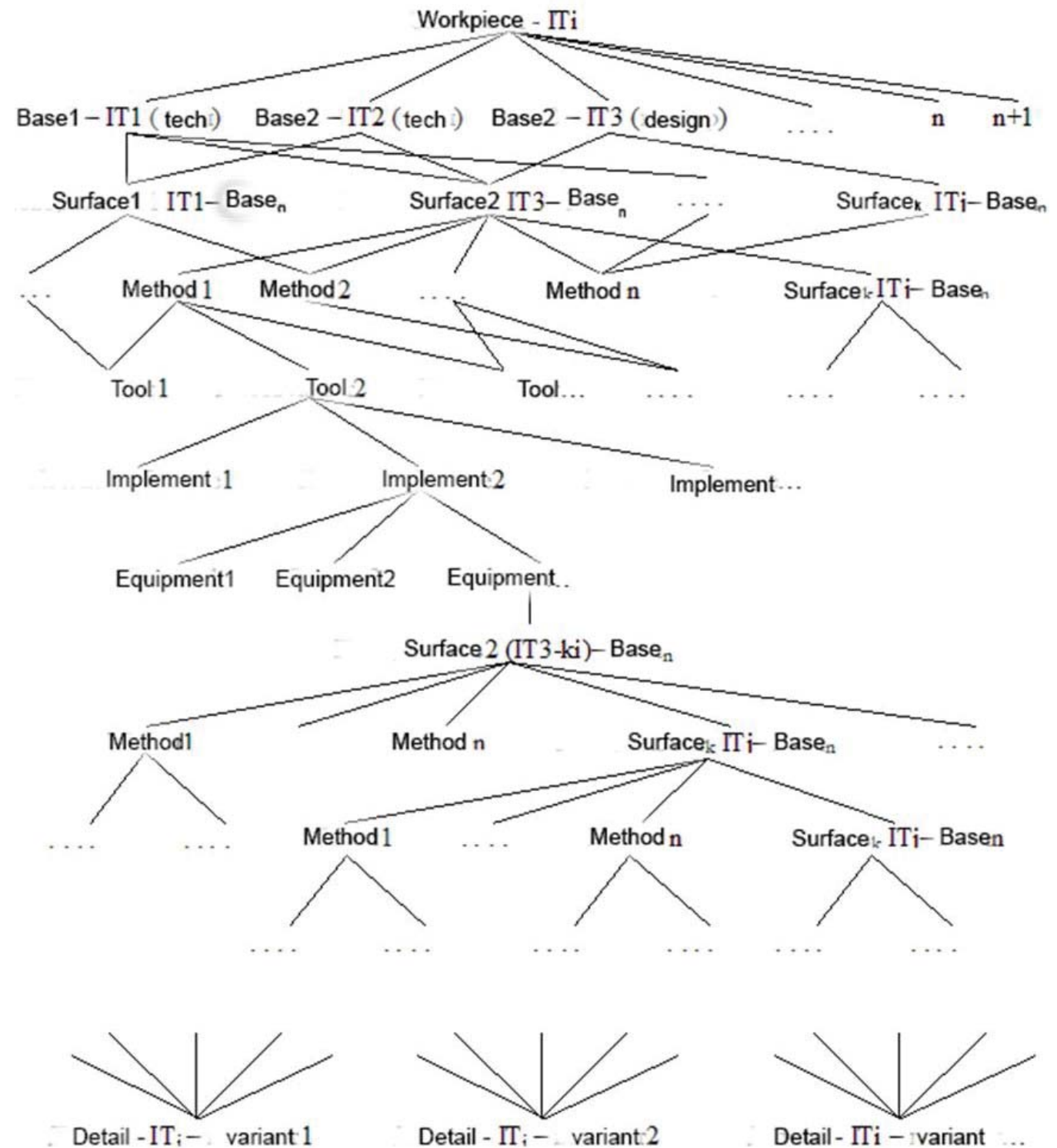

Figure 2: Graph of variant development for machining plans

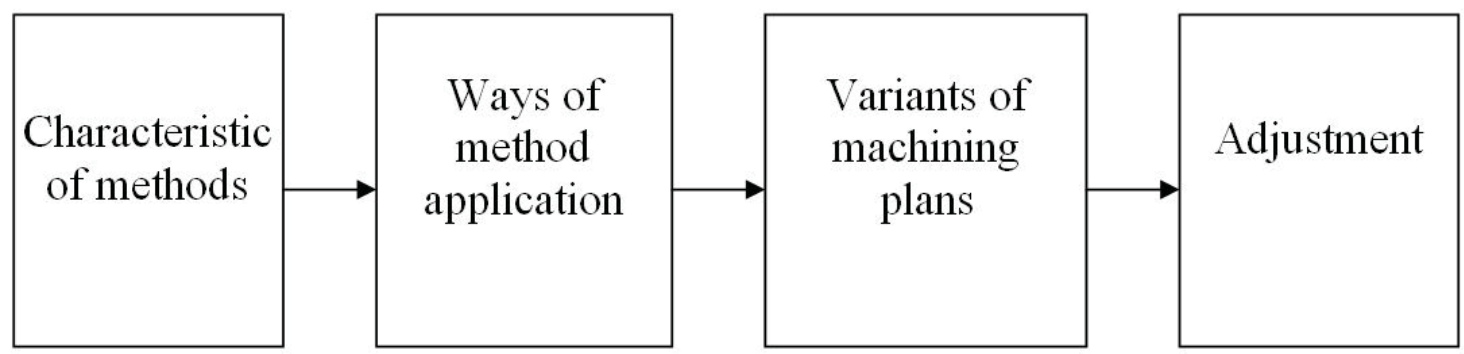

Figure 3: Database for machining planning 
In view of the fact that the processed surfaces contain several dimensions and these dimensions lie in the planes with having surface errors which need to be corrected by the corresponding method of machining, characteristics of these methods will be divided into the characteristics corresponding to machining of the dimensions in the particular plane.

To determine a quality class of transition in the machining plan, the mathematical model displaying machining process, namely, increase in a quality class of machining is used. This model is based on the statement that when roughing quality class increases 3,4 units, semi-finishing $-1,2$ units, finishing -1 unit, smoothing -0 units. As machining overlap for the choice of a cutting depth method, is calculated by a technique where calculation is carried according to the machine part dimension, so the mathematical model of machining quality-class search will be based not on specification (increase) of a quality class, but on roughening, that is the direction of machining calculation will be carried from a detail surface.

As a result, the system gives the maximum quantity of possible variants of machining plans sorted by increase in accuracy of machining. For compilation of the maximum number of variants the database is used (a Figure 3 and an algorithm, a Figure 4).

The table submitted by the block 1 is used for description of method characteristics of machining. The name of the method, designation of dimensions, describing a particular surface, the minimum and maximum value of the dimensions of ranges from a geometrical row are entered in this table. In view of the fact that one method can be used to machine surfaces with various dimension

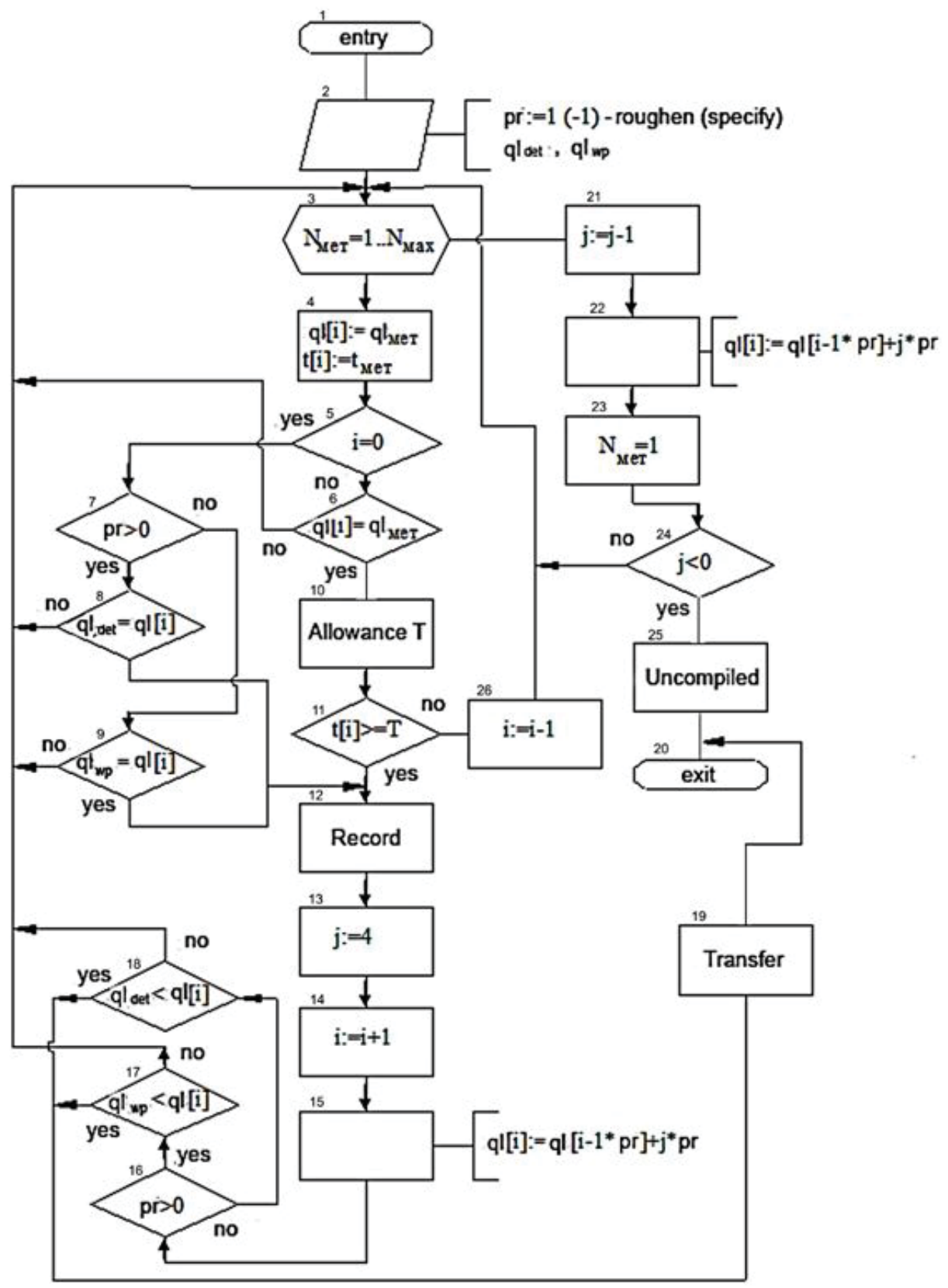

Figure 4: Algorithm of machining planning 
descriptions (various number of dimensions), the maximum quantity of possible directions (designations) of dimensions (the maximum quantity of the directions) is included into the description of a method.

Quality class of machining is entered further, namely, clearance, the maximum accuracy of profiles (even if there are several of them for one direction of dimension), a roughness (minimum), depth of a defective layer (minimum), maximum depth of cutting. These are the generalized data of characteristics of the tools belonging to this method of machining.

The same method can be applied by various means, namely, multi spindle machining, multi tool machining, machining by the combined tool, machining by the compound tool.

Therefore, the table of possible means of machining method application is created for this purpose. This table is provided by the block 2 . It is necessary to enter the name of a machining method, means of machining, an application method, what dimension is to be corrected: distance or a surface, dimension designation according to a surface (for the surface dimensions) and designation of a placement in relation to the surface dimension (for the distance dimensions).

The table provided by the block 3 is made to record plan variants. The table contains the following data: the number of a plan variant, the number of a surface, the name of a machining method, a quality class of machining, cutting depth, and an overlap.

The problem of machining planning can be solved on the basis of the algorithm represented in the figure 4 .

In the block 2, it is necessary to enter the following data: machining limits (the quality class of a machine part and the quality class of a workpiece), the number of steps allowing to remove overlap. Design will be conducted by the roughening method or the method of quality class adjustment. The block 3 represents the table where data on machining methods are stored. In the block 4, values of method quality class and cutting depth are bound to variables of the quality class amount and cutting depths.

The search of a necessary method in the quality class table is run by means of blocks 8 and 9 . In the block 10 , the machining overlap is calculated and compared to cutting depth of a current method (the block 11).

In case of unsatisfactory result, the search of another method is run in the block 3 . In case of affirmative answer, the position of quality class amounts (block 11) and depths (block 13) increases. In the block 15, calculation of a quality class is carried out from the formula:

$q_{l}[i]=q_{l}[i \pm 1 \times m]+j \times m$

where $q_{1}[i]$ - a machining quality class at this stage;

$q_{1}[i \pm 1]-$ a quality class at the following stage of machining $(+)$, a quality class at the previous stage of machining $(-)$.
In the block 12, a record of method parameters is made in the table of transitions.

In the block 13, it is set that roughening (adjustment) will be made by 4 units. Calculation process continues before it reaches a workpiece (block 17) or machine part (block 18) quality class. Then transfer of machining plans is made (block 19).

If the method for overlap machining is not in the table, then it is set in the block 21 that roughening (adjustment) will be made 1 unit less and method search continues from the beginning of table (block 22). The result given by the block 22 leads either to a further method search, or to a message that the machining plan cannot be developed (block 23). The hitting a roughening (adjustment) unit of a negative value is a basis of the given result.

Herewith it is taken into account that the machining plan for a surface, which dimension enters the range of dimensions, which in turn includes surface dimension of the same type and class with developed machining plan, is not developed, and the developed plan is taken for the surface it is not made for. Thus before machining plan developing, a search of a surface of the same type and class (with the identical subrange) takes place in the table of the machine-part surface description. The search of the plan in the table of variants is conducted according to a number of a found surface, in case of plan availability it is being borrowed, in case of its absence a plan is being developed.

Final development will occur only after selection of all PP elements while steps are being developed.

If physical-mechanical parameters of a workpiece differ from those of a machine part, then it is necessary to enter non mechanical methods of machining for achievement of the set qualities. As these methods of machining suppose hardening of a blanket of a particular surface or a machine part in whole, the data of steps need to be entered in the end of machining. As they worsen other parameters, this non mechanical step is entered next to the last and recalculation of overlap with checking of a machining possibility by the next method is made. Herewith perhaps it will be necessary to add additional step. Hardness of a surface is not taken into account (block 3 ). Other non-mechanical steps are inserted after step forming in the dialog mode.Thus, the user is able to conduct an effective analysis of a large amount of information, including in a real-time environment [2].

\section{THE DEVELOPMENT OF CADS FOR PRODUCTION PROCESSES OF COMPONENT PART MACHINING FOR SINGLE-PIECE PRODUCTION AND REPAIR CONDITIONS}

Two variants of plan development for surface machining are possible during designing. The first variant implies their automatic selection, offering the choice of an optimal variant by the technologist. Various variants of methods of workpiece machining to a state of machine part are given to the technologist after automatic calculation. 
Information on a machine part is divided into information (Figure 5):

- graphical [3-5];

- on material;

- on hardness;

- on technical requirements $[5,6]$;

- on surface geometry;

- on surface dimensions;

- on the accuracy of a form of surfaces;

- on coordination of a surface in space and in relation to a workpiece surface;

- on placement accuracy.

All parameters of the offered plan versions are being received by calculation. The technologist can choose a final version on the basis of their experience. Hereby there is a possibility of the received variant adjustment. However, the technologist can give the choice of a final version of machining plans to the program.

The second variant of the choice of machining plans implies their preliminary choice and parameter calculation without the program $[7,8]$. Thus, information on machining plans is to be entered if their calculation is not made by the program.

For convenience of machine-part parameter input the technologist can open the machine part drawing in the given program, but it is necessary to enter the data manually. The program does not read off the data from the machine part drawing.

In the process of information input, the technologist enters data on machine part material, its hardness and the state of a blanket. Then they select a machine part surface with its number indication according to the graphics image. The machine part is being divided into elementary surfaces starting with the left end face or the side shown on the design drawing. Hereby all surfaces without their division into different types and classes go through numeration, i.e. a graphics image corresponds to a particular type and a class of a surface - cylindrical, inside or outside, etc. Then dimension characteristics of a surface are entered, namely, the dimensions through parameters and their values, their accuracy - a quality class and the main displacement, or directly the upper and lower displacement and surface roughness. If there are general surface requirements on the machine part drawing, namely dimension accuracy and roughness, these parameters and their values are to be specified for each surface. If there is not a necessary surface in the program database, the technologist can add it into the appropriate tables without program code change.

The menu is made dynamic in order to enter parameters and their values for different types and classes of a surface. It provides the use of only one window in the software. Hence, the structure of software becomes simpler and additional adjustment of a program code is not required in case if the menu for data input on a particular surface is not suitable.

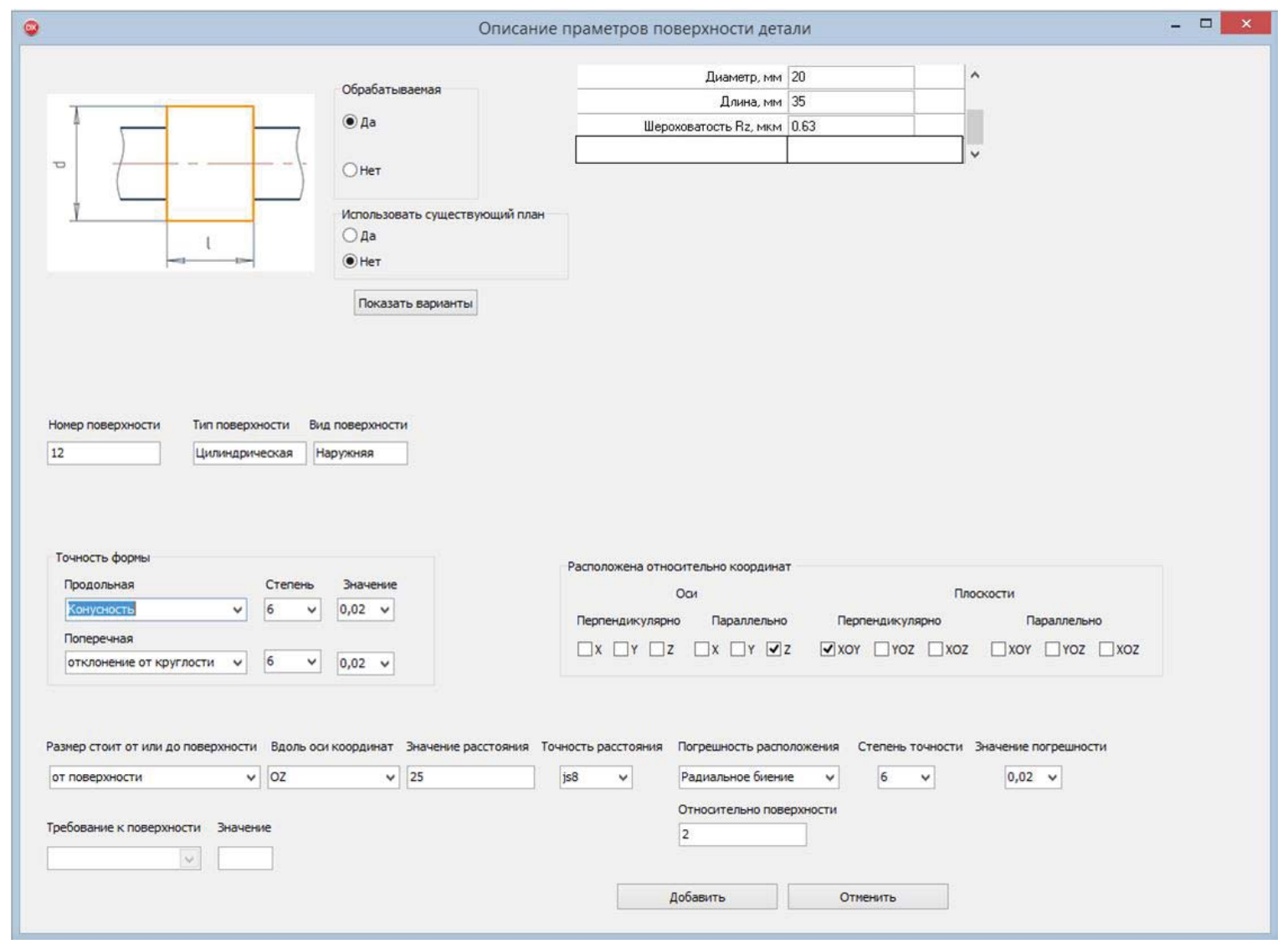

Figure 5: The window of machine-part parameter input 
Then the accuracy of forms in the longitudinal and cross direction, their designation, degree of accuracy and value are specified. Software has internal information in the form of databases therefore tabular data can be entered automatically while entering the determining data, i.e. when specifying accuracy degree for the determined dimensions and the types of deviation, values of these deviations are removed automatically. The closest degree of accuracy will be determined by the entered values of accuracy.

Surface orientation in relation to coordinate axes and planes is entered for surface coordination. The information on the coordinating dimensions (their values, accuracy, placement in relation to coordinate system) is entered as well. The coordinate system is determined in advance and is guided according to technological preferences. When entering the distances orienting the given surface, it is necessary to pay attention to the circumstance that only surfaces of a shaped form have own dimensions, as well as cylindrical surfaces, flat surfaces have dimensions which are coordinating distances at the same time. Also it is necessary to enter information on placement errors.

Information on additional machine part requirements is entered to the respective fields and is considered in case of determination of stages and methods of machining, calculation of the cutting modes, calculation method of allowances [9].
Information on workpiece is divided into information:

- graphical;

- on material;

- on production method;

- on surface geometry;

- on surface dimensions;

- on the accuracy of a surface form;

- on coordination of a surface in space and in relation to the workpiece surfaces;

- on placement accuracy.

In the Figure 6, the window of workpiece parameter input is given.

When designing, two variants of a workpiece choice are possible. The first variant implies its automatic choice where the technologist can choose an optimum method of its production. Various variants of methods of work piece production for the given machine part are provided to the technologist after automatic calculation. All parameters of the offered work pieces are received by calculation. Preliminary economic calculation of cost on the work piece mass is also provided. The technologist can choose a final version on the basis of their experience. Hereby there is a possibility of the received variant adjustment.

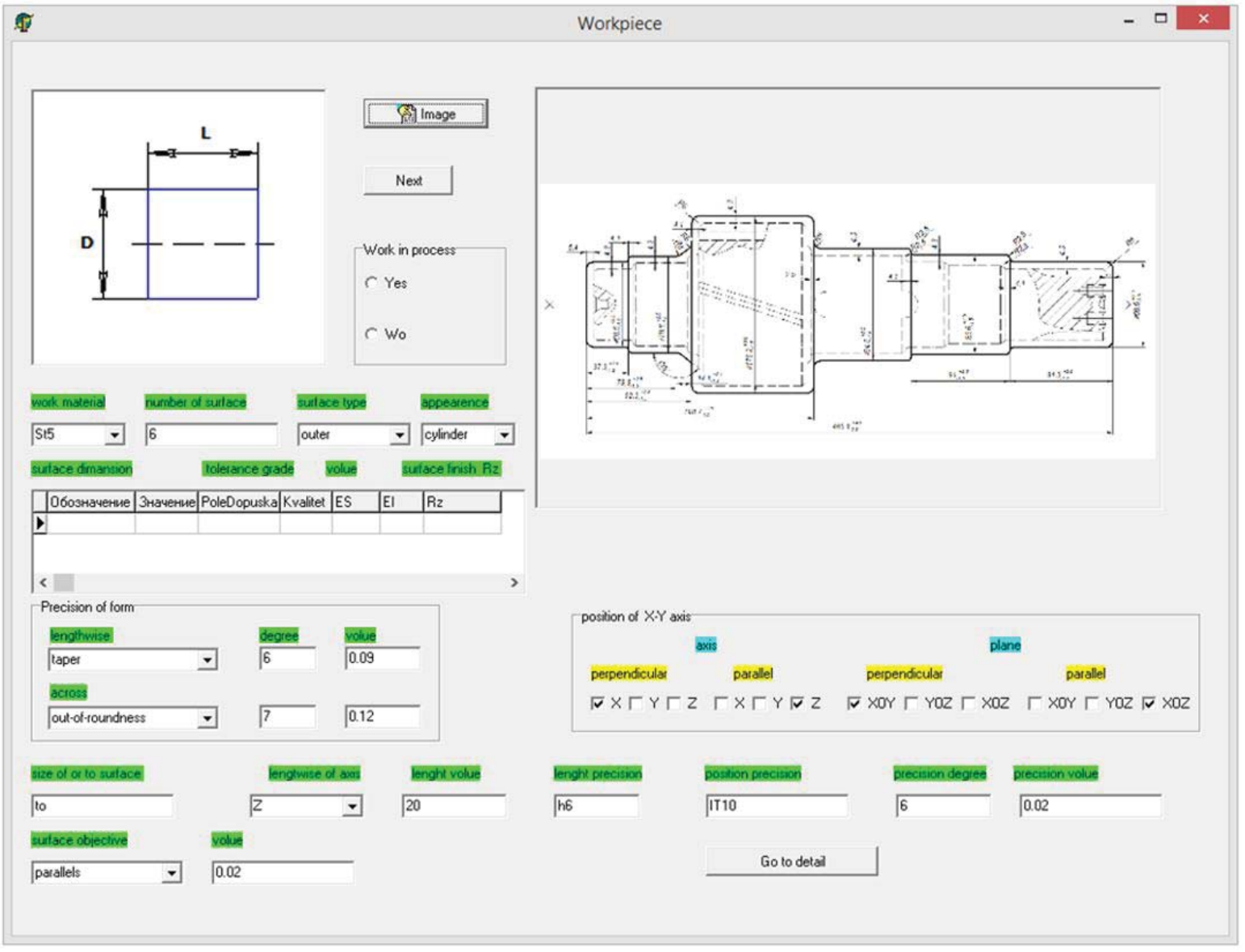

Figure 6: Window of workpiece parameter input 
The second variant of the workpiece choice implies the preliminary choice of the method of workpiece production and calculation of its parameters without the program. Thus information on the workpiece is to be entered if its calculation is not carried out by the program. In case of the automatic choice of a workpiece and automatic calculation of its parameters, the program enters data according to internal calculations. Information on the workpiece surfaces is entered on the basis of information on surfaces of the processed machine part. The workpiece is divided into as many surfaces as a machine part is. Hereby it is necessary to consider that the neighboring surfaces of a machine part having the same diameter on the workpiece after overlap addition do not merge in one surface on the workpiece, and have the same numbers as the machine part does.

For convenience of workpiece parameters input the technologist can open the workpiece drawing in this program but it is necessary to enter the data manually. The program does not read off the data from the workpiece drawing.

In the process of information input the technologist enters data on workpiece material, its hardness and the condition of a blanket. Then using the graphics image the technologist chooses the surface of a workpiece with its number indication according to number given on the machine part. A particural type and the class of a surface (cylindrical, internal or external, etc.) correspond with the graphics image. Then surface dimensional characteristics, actually the dimensions through parameters and their values, their accuracy are entered (a quality class and a major deviation, or directly the upper and lower deviation, and surface roughness. When specifying on the drawing of workpiece of general requirements to surfaces, namely the accuracy of the size and a roughness, these parameters and their values shall be specified for each surface. If there are general surface requirements on the workpiece drawing, namely dimension accuracy and roughness, these parameters and their values are to be specified for each surface.

The menu is made dynamic in order to enter parameters and their values for various types and classes of a surface. It provides the use of only one window in the software. Hence, the structure of the software becomes simpler and additional adjustment of a program code is not required in case if the menu for data input on a particular surface is not suitable.

Then the accuracy of forms in the longitudinal and cross direction, their designation, degree of accuracy and value are specified. Software has internal information in the form of databases therefore tabular data can be entered automatically while entering the determining data, i.e. when specifying accuracy degree for the determined dimensions and the types of deviation, values of these deviations are removed automatically. The closest degree of accuracy will be determined by the entered values of accuracy.

Surface orientation in relation to coordinate axes and planes is entered for surface coordination. The information on the coordinating dimensions (their values, accuracy, and placement in relation to coordinate system) is entered as well. The coordinate system is determined in advance and is guided according to technological preferences. When entering the distances orienting the

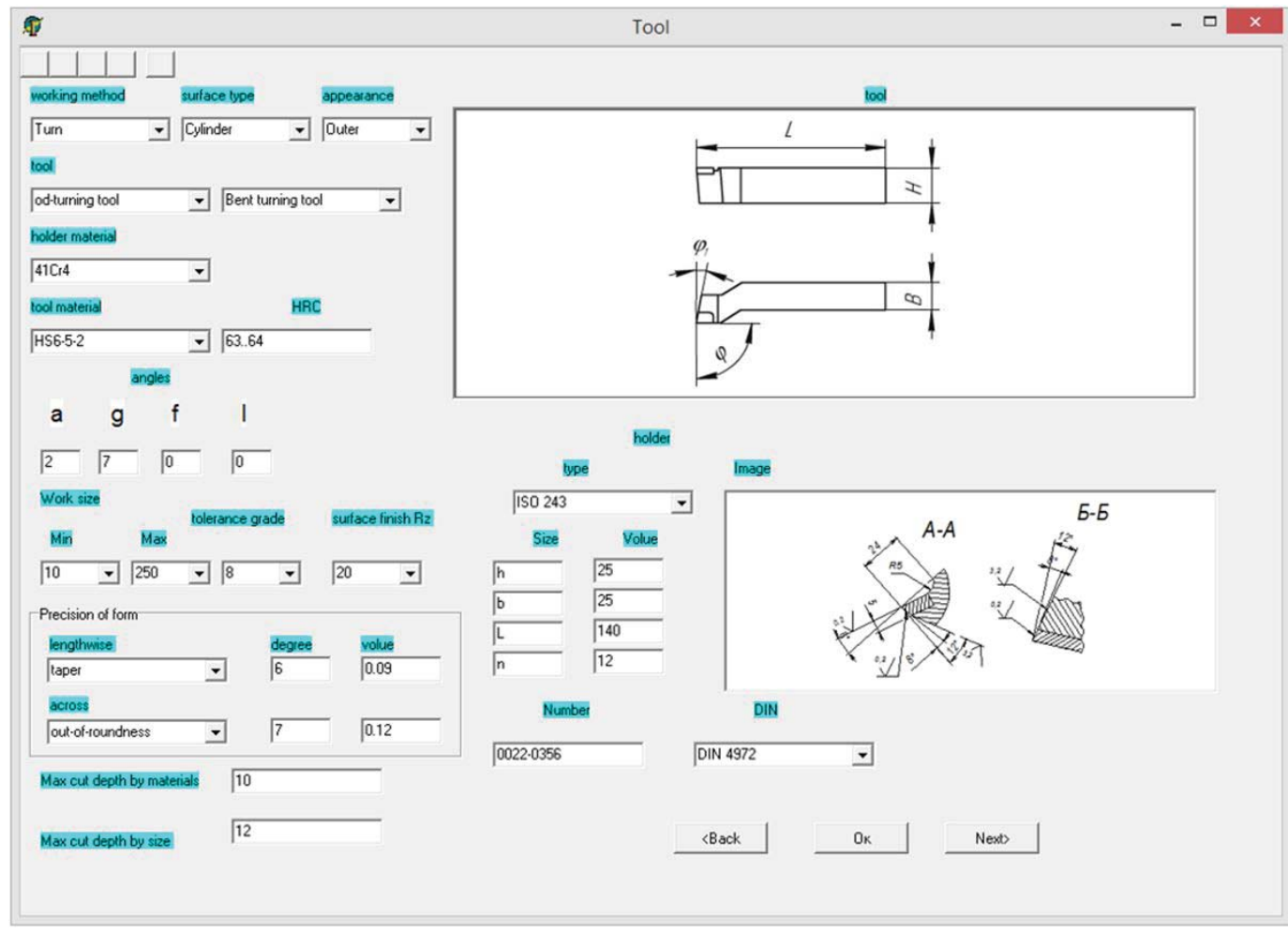

Figure 7: Window of tool parameter input 
given surface, it is necessary to pay attention to the circumstance that only surfaces of a shaped form have own dimensions, as well as cylindrical surfaces, flat surfaces have dimensions which are coordinating distances at the same time. Also it is necessary to enter information on placement errors.

In the Figure 7 , the window of tool parameters input is given.

For the use of the tool, information on the surface of the machined workpiece and a machining method for the use of the given tool is entered in the software, which provides its communication with the corresponding elements of production process, namely:

- graphical;

- $\quad$ on a machining method;

- $\quad$ on the type of the machined surface;

- $\quad$ on the placement of the machined surface.

The entered information includes information on material of the cutting part of the tool, its hardness. Angles of the cutting part of a tool (definite for each type and a class of the tool) are entered in order to produce a workpiece of the defined geometry and eventually a machine part, to make a calculation of the cutting modes, calculation method of allowances .[9].

Information on a tool holder is entered as well. This information includes a type of a holder and its standard, holder material, its dimensions.

It is necessary to have information on power abilities of the tool to provide the maximum machining performance preserving working parameters of the tool. For this purpose, information on physical-mechanical properties of material of a holder and a cutting wedge is entered. Also the data on the maximum dimensions of a removing material layer and the tool dimensions are entered. It is also necessary to enter the data on tool hardness.

The data on a number of the tool according to the standard and respectively a number of the standard are considered to be necessary information.

In order to choose a particular tool for production of defined properties of the machined surface, it is necessary to provide information on the maximum possible accuracy, which can be received using this tool. For this purpose, a quality class of accuracy of the given surface, roughness, accuracy of the given form in the longitudinal and cross direction (designation of deviation, degree of accuracy and its value)

Finally, it is required to carry out an economic assessment of the chosen tool. Therefore, the data on tool cost are also entered.

The use of the tool in production conditions implies its stock-taking. Information on its nomenclature number, quantity and wear and tear is required for this purpose. In addition, the possible quantity of resharpenings, as well as quantity conducted is taken into account in technological preparation of production.
The menu is made dynamic in order to enter parameters and their values for different types and classes of a tool. It provides the use of only one window in the software. Hence, the structure of software becomes simpler and additional adjustment of a program code is not required in case if the menu for data input on a particular tool is not suitable.

Information on the tool is to be entered before its use in the software. The graphic information from the image of a cutting tool or its geometry is not read off, it is necessary to enter it manually in the appropriate fields.

Information can be entered from information arrays of a particular enterprise, via the appropriate interface (it is not given in the thesis) for convenient storage and use of data on the tool. Hereby additional data processing is required for what the software suggests a user to fill in missing data, controlling them automatically.

In this way, information is entered for equipment and implements (Figures 8, 9). Further automatic design of production process of machine part manufacture is made with information transfer to particular working places.

Thus developed models provide the solution of the main technological problems as follows:

1. determination of machine-part production stages;

2. determination of schemes of its basing, a set of bases and an order of their preparation;

3. forming of schemes of technological dimensional chains;

4. determination of methods of step implementation, types of the equipment and tool;

5. determination of a structure of technological steps;

6. distribution of overlaps on steps;

7. calculation and recalculation of interoperational dimensions;

8. determination of properties and parameters of the tool, implements, machines.

The developed technique of production design in the non interactive mode does not require high qualification of the technologist and reduces amount of errors in the development of production processes. In order to form input data, knowledge of production processes of analogous machine parts or products production is not required.

The designed system is characterized by properties of universality, multifunctionality, complexity that gives the chance of its use in modern multiproduct production.

As part of the scientific research implementation, a program "Development of the Kazakhstan system for automating the technological preparation of engineering production" was developed and a certificate was received on entering information into the state register of rights to objects protected by copyright No. 4522 of July 15, 2019.

This program has been tested at the Almaty Heavy Engineering Plant (Almaty, Kazakhstan). The proposed program was easily adapted to existing production, as the 


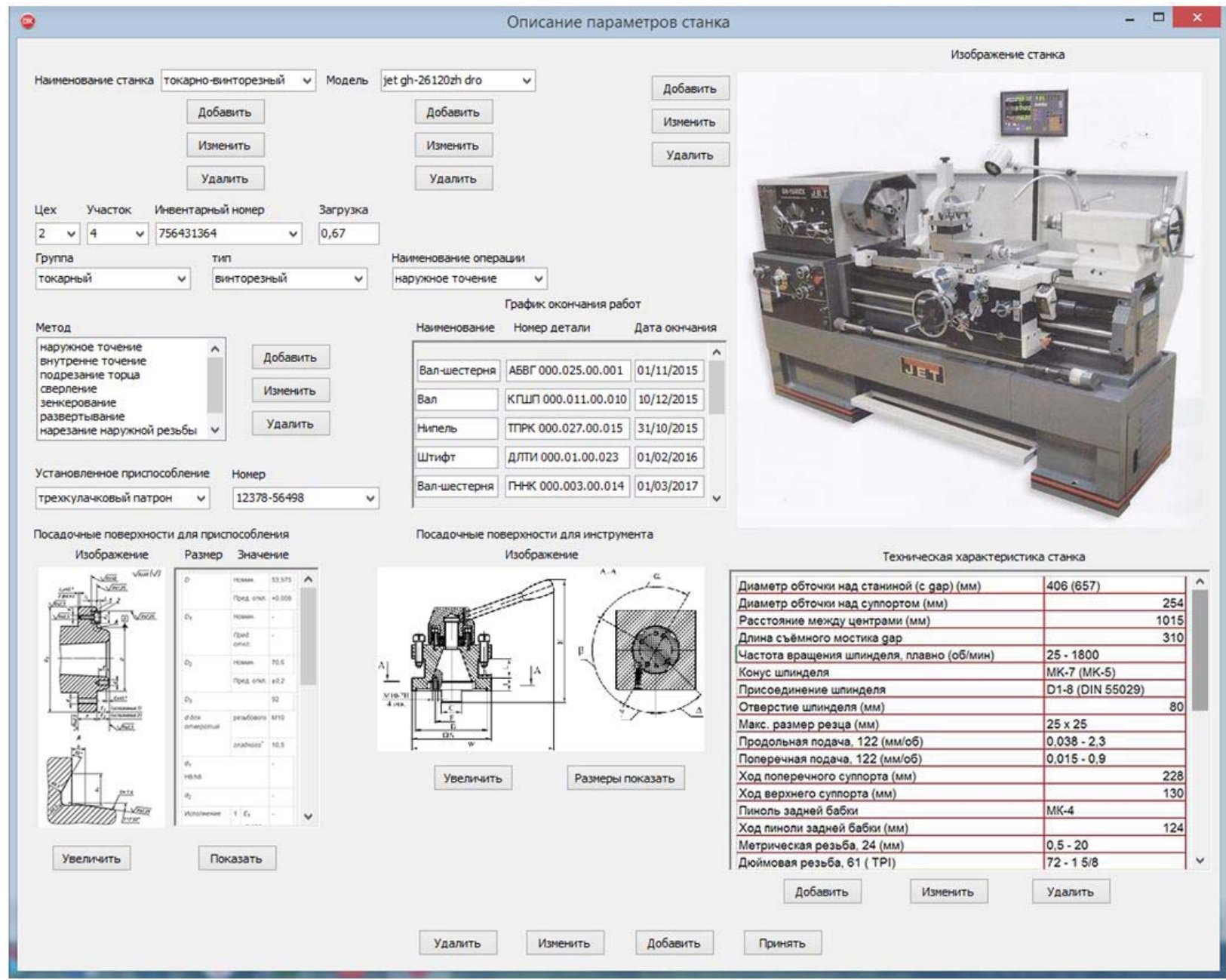

Figure 8: Window of machine parameter input

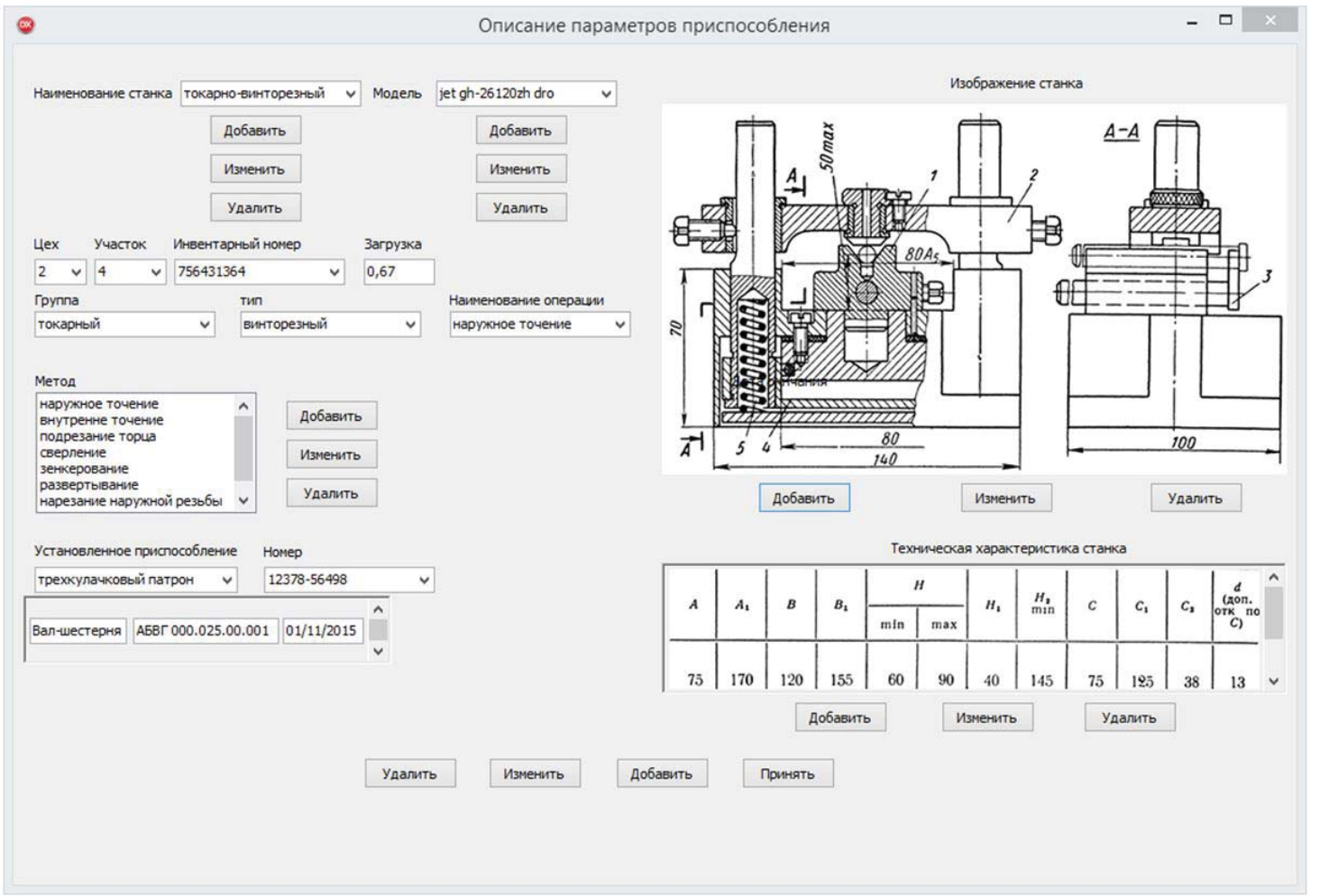

Figure 9: Window of implement parameter input 
choice of hardware was based on the minimum system requirements.

The implemented program has shown its effectiveness in the development and design of technological processes. This led to a reduction in technological time for design, the elimination of subjective errors and lower production costs while increasing the volume of output information.

\section{CONCLUSION}

1. The use of a developed technique of production design in the noninteractive mode requires the technologist of low qualification, errors in the development of production processes are reduced to a minimum. In order to form input data, knowledge of production processes of analogous machine parts or products production is not required.

2. The designed system is characterized by properties of universality, multifunctionality, complexity that gives the chance of its use in modern multiproduct production:

- allows to make technology solutions at the objective level that excludes errors when designing,

- $\quad$ gives a chance to develop production processes for machine parts which were not produced at the given plant earlier, that is when there is no opportunity to use standard or group engineering procedure as an analog,

- $\quad$ is applied to any types of machine parts,

- is used for any production processes,

- can be adjusted for any type of production: repair, single, small-scale, medium-scale, large-scale, mass.

3. The developed software is universal, allows entering information for various objects, without a program code change. For this purpose, menu is made dynamic. It provides the use of only 15 windows for corresponding information input in the software. Hence, the structure of software becomes simpler and additional adjustment of a program code is not required in case if the menu for data input on particular element of process medium is not suitable. For convenient storage and use of data on process medium, information can be entered from information arrays of a particular enterprise

4. Developed models and technique allowed to increase amount of output information to the level of operational flow charts that allows to reduce piece and calculation time of machining 1,2-1,4 times, to cut consumption rate of machined material by $1,3-$ 1,5 times.

5. Experimental research of design process showed that labor input of production process development for machine parts of "Axis", "Lever" and "Cogwheel" type was reduced by $3-4$ times, for machine parts of "Case" type - by $4-5$ times.

\section{REFERENCES}

1. Schaede, C., Seifermann S., Metternich, J. (2018). Automated generation of CNC programs for manufacturing of individualized products. 51st CIRP Conference on Manufacturing Systems Procedia CIRP 72, p. 1251 - 1257

2. Koltunov, I., Panfilov, A. V., Poselsky, I. A., Chubukov, N. N., \& Matkov, S. S. [2018]. Review of the big data technology use in the medical prognosis. Journal of Applied Engineering Science, 16(4), 553-560.

3. Illyustrirovannyiy opredelitel detaley obschemashinostroitelnogo primeneniya: rukovodyaschiy tehnicheskiy material. Klassyi 40 i 50. Obschesoyuznyiy klassifikator promyishlennoy i selskohozyaystvennoy produktsii (Moscow: Izdatelstvo standartov) p. 238.

4. Tehnologicheskiy klassifikator detaley mashinostroeniya i priborostroeniya. Moscow: Izdatelstvo standartov. Vol. 1 - p 100; Vol. II - p. 84.

5. Yakushev, A.I., Vorontsov, L.N., \&Fedotov, N.N. (1986). Vzaimozamenyaemost, standartizatsiya i tehnicheskie izmereniya. Moscow: Mashinostroenie. P. 352.

6. Myagkov, V.D. (1998). Dopuski i posadki: Spravochnik. Leningrad: Mashinostroenie. Vol. 1.P. 544.

7. Kosilova, A.G., Mescheryakov, R.K., \& Kalinin, M.A. Tochnostobrabotki i pripuski $v$ mashinostroenii: Spravochniktehnologa. Moscow: Mashinostroenie. P. 288.

8. Kosilova A.G., Mescheryakov R.K. (1985) Spravochnik tehnologa-mashinostroitelya. Moscow: Mashinostroenie. P. 656.

9. Kovan V.M. (1987) Raschet pripuskov na obrabotku v mashinostroenii. Moscow: Mashgiz. P. 304.

Paper submitted: 25.04.2019.

Paper accepted: 13.08.2019.

This is an open access article distributed under the CC $B Y-N C-N D 4.0$ terms and conditions. 